\title{
Evaluation by Point of Care Ultrasound in Patients with Sepsis
}

\author{
Zheng Xin, Xiong Ying, Wang Ni, Su Yang, Zhang Yanjun* \\ Department of Anesthesiology, The Second Affiliated Hospital of Dalian Medical University, Dalian, China \\ Email address: \\ zhengxinxin008@163.com (Zheng Xin), xiongyingxy1001@126.com (Xiong Ying),731854418@qq.com (Wang Ni), \\ ocean107703@163.com (Su Yang), zytdsz@hotmail.com (Zhang Yanjun) \\ *Corresponding author
}

\section{To cite this article:}

Zheng Xin, Xiong Ying, Wang Ni, Su Yang, Zhang Yanjun. Evaluation by Point of Care Ultrasound in Patients with Sepsis. International Journal of Clinical Oncology and Cancer Research. Vol. 6, No. 2, 2021, pp. 69-73. doi: 10.11648/j.ijcocr.20210602.13

Received: March 22, 2021; Accepted: April 23, 2021; Published: April 30, 2021

\begin{abstract}
At present, point of care ultrasound (POCUs) is widely used in emergency and critical care disciplines, to realize the rapid diagnosis and evaluation of critical diseases such as dyspnea and shock. The rapid diagnosis and evaluation of critical diseases such as dyspnea and shock can provide a good reference for the development of anesthesiology and a rapid diagnostic tool for the perioperative critical diseases of respiratory or circulation disorders. Point of care ultrasound has the characteristics of real-time, dynamic, non-radiation and repeatability, so it has a good advantage in perioperative application. provide visual evidence for treatment, and promote perioperative treatment closer to the direction of evidence-based medicine. Point of care ultrasound has many simple and effective protocols for the diagnosis of critical diseases. The corresponding procedures can be selected according to different symptoms, and different procedures can also be combined to achieve the comprehensiveness and accuracy of the assessment, avoid missing important information, especially for emergency patients. Perioperative pulmonary edema leads to hypoxemia, etiology can be quickly identified and dynamically evaluated by point of care ultrasound, B-lines detected by lung sonography are signs of pulmonary interstitial syndrome. Point of care gastric ultrasound can effectively and reliably evaluate the nature and volume of gastric contents, and its clinical application can be extended to the evaluation of gastric contents in patients with delayed gastric emptied emergency, thus reducing the risk of perioperative reflux aspiration, point of care ultrasound provides visual evidence for perioperative decision making, ensure patient safety and improve outcomes.
\end{abstract}

Keywords: Point of Care Ultrasound, Perioperative, Pulmonary Interstitial Syndrome, Gastric Content

\section{Introduction}

Point of care ultrasound is widely used in emergency and critical care medicine, and the guidelines further promote its use [1-4].

Perioperative point of care ultrasound is one of the most important application areas [5], but it is mostly limited to ultrasound-guided nerve block and vascular puncture at present time, point of care gastric ultrasound can evaluate the nature and volume of gastric contents [6].

Point of care ultrasound has been used in perioperative period for more than 30 years and is a powerful tool for perioperative diagnosis and treatment evaluation [7].

The BLUE protocol of bedside ultrasound is used to evaluate patients with hypoxemia, while the FATE protocol can quickly evaluate cardiac function and the RUSH protocol can determine the cause of shock, which is simple and efficient [1].

The condition of emergency patients is complex and changeable, and the movement is difficult, so it is necessary to quickly identify the cause and timely treatment, point of care ultrasound is an efficient tool. Lung aspiration is a serious perioperative complication, and aspiration of gastric contents is a common cause.

Under the same physiological state, the higher the gastric content volume, the more likely it is to reflux and lead to aspiration.

In particular, a large number of solid particles and low $\mathrm{pH}$ liquid inhalation.

Perioperative pulmonary aspiration can not be completely 
avoided according to the current preoperative fasting guidelines, gastric emptying is delayed in long-term bed bed, elderly patients, diabetes patients and obese patients, and routine fasting time cannot guarantee complete gastric emptying.

The risk of aspiration during perioperative period mainly depends on clinical experience. Practical and non-invasive bedside test technique is an important guarantee for the safety of clinical anesthesia. Different groups such as pregnant women gastric emptying situation is very different, increasing the difficulty of judging gastric emptying Pulmonary aspiration of gastric content is a serious anaesthetic complication that can lead to significant morbidity and mortality [8]. Gastric emptying is delayed in certain populations, such as trauma, diabetes, and pregnancy, and routine gastric emptying times may be inaccurate, leading to inadequate assessment of the risk of gastric content reflux aspiration. The author evaluated perioperative sepsis patient by point of care ultrasound, found full stomach and acute lung injure, and the report is as follows.

\section{Case Presentation}

The patient, male, 52 years old, was admitted to the hospital in the green channel of intestinal obstruction in the emergency department, with a gastrointestinal decompression tube, and no laboratory examination was conducted.

Vital signs in the operating room were as follows: blood pressure $120 / 70 \mathrm{mmHg}$, heart rate 154 times / $\mathrm{min}$, SpO 94\%, body temperature $38^{\circ} \mathrm{C}, 28$ times /min of respiration, and clear consciousness.

Point of care ultrasound was used for supine scanning, and lung ultrasound was performed according to Blue protocol. Multiple B-lines were found in both lungs.

Scanning of gastric antrum showed a cross-sectional area of $3 \times 3 \mathrm{~cm}$, ultrasound estimate the volume of gastric fluid as follow [9].

\section{$\mathrm{GV}(\mathrm{ml})=27.0+14.6 \times$ right-lat $\mathrm{CSA}-1.28 \times$ age}

It is applicable to adult, can predict volumes more than 200 $\mathrm{ml}$.

At present, the patient developed acute pulmonary edema, which is an acute lung injury secondary to sepsis, combined with satiety, and also full stomach.

After the gastrointestinal decompression tube was fully attracted with a negative pressure aspirator, oxygen was inhaled at $6 \mathrm{~L} / \mathrm{min}$, and rocuronium was used for rapid endotracheal intubation. No reflux or pulmonary aspiration occurred.

Intraoperative intravenous administration of methylprednisolone and ambroxol to inhibit pulmonary inflammation.

Tumor obstruction was found intraoperatively, and colostomy was performed. After surgery, the patient was sent to the ICU ward. After 36 hours of mechanical ventilation support, the ventilator was withdrawn.

\section{Discussion}

Patients with sepsis are prone to lung injury due to over-activated inflammatory response, and even progress to acute respiratory distress syndrome [10], which is difficult to treat.

Early clinical identification, diagnosis and treatment can improve the prognosis of patients $[11,12]$.

Pulmonary ultrasound is no less than CT in the recognition of pulmonary edema, pneumothorax, pleural effusion and other pulmonary lesions [13], and can detect pulmonary edema earlier than clinical symptoms [14], especially suitable for operating room and intensive care unit and other inconvenient mobility conditions. Pulmonary ultrasound allows rapid visualization of lung lesions at the bedside, with each sign derived from the physiological and pathophysiological nature of the lungs.

Simple ultrasound equipment can be completed lung ultrasound examination;

Lung is a mixture of liquid and gas, and when the ratio of liquid and gas changes, different ultrasonic signs will be produced.

Acute pulmonary disords often accumulates into the pleura, which can be detected by pulmonary ultrasound [13].

Pulmonary ultrasonography showed normal pleural slide sign on line $\mathrm{A}$, line $\mathrm{B}$, line $\mathrm{B}$, diffuse interstitial syndrome, consolidation and effusion, and no pleural slide sign on line $\mathrm{A}$ or lung points.

Because of the real time dynamic advantage of lung ultrasound, it can further promote the deep understanding of lung pathophysiological changes.

The most common causes of patients with mechanical ventilation include ARDS, pulmonary infection, acute exacerbation of chronic obstructive pulmonary disease, etc., with a variety of lesions, especially during the treatment process may change at any time, routine pulmonary ultrasound can be timely assessed including the status of changes in lesions, and even the occurrence of pneumothorax.

Pulmonary ultrasound examination has been gradually standardized in intensive care unit as a tool for visual medicine [13], it is an important part of severe ultrasound, and can be used for early recognition and treatment of acute pulmonary dysfunction including ARDS, so as to improve the outcomes [2]. It is recommended that severe patients receiving mechanical ventilation be routinely performed pulmonary ultrasound examination.

Pulmonary ultrasound should give priority to the evaluation of pleural lines [13].

Pulmonary ultrasonography originated from pleura and is the basis of pulmonary ultrasonography.

The pleural line is the junction of soft tissue (fluid rich) chest wall and lung tissue (gas rich), the lung-chest wall junction.

The pleural line acts as a mirror, 'mapping' the different lesions in the lungs onto the ultrasound probe, the pleural line is usually visible unless there is substantial subcutaneous emphysema. 
In lung ultrasound, the pleural line is accurately located to distinguish between intrapulmonary lesions and lesions in the pleural cavity or subcutaneous soft tissue.

The most important thing to evaluate is the presence of pleural slide sign, except for the possibility of pneumothorax or local lack of ventilation. It should be noted that in patients with pleural adhesion or lung consolidation, there may be absence of pleural slide sign.

And pleural thickness and the smoothness of the pleura is also to a certain extent, prompt diagnosis of some diseases, such as pleural adhesion, generally will be appeared pleural thickening performance, such as the lungs are filled B line of smooth pleura lines suggest the possibility of acute lesions, matte pleural line prompt more chronic lesions or pulmonary fibrosis, etc.

Acute respiratory failure bed lung ultrasound diagnosis process (bedside lung ultrasound in emergency, BLUE) within 3 min in the lungs and rapid screening of deep vein thrombosis, about $90.5 \%$ of the causes of acute respiratory failure or hypoxemia make rapid and accurate diagnosis, including increased hydrostatic pulmonary edema, chronic obstructive pulmonary diseases acute exacerbation or severe bronchial asthma and pneumonia, pulmonary embolism, pneumothorax.

In recent years, a number of studies have found that pulmonary ultrasound-BLUE procedure alone has certain limitations in the assessment of the etiology of acute respiratory failure.

The modified BLUE and MBLUE procedures can significantly increase the sensitivity, specificity, and accuracy of lung consolidation and atelectasis detection in ICU patients.

Studies have found that the diagnostic accuracy, sensitivity and specificity of integrated cardiopulmonary ultrasound for cardiogenic pulmonary edema and pneumonia are higher than that of single pulmonary ultrasound, while there is no significant difference in the sensitivity and specificity of pulmonary embolism and pneumothorax.

Therefore, intensive ultrasound can be helpful in rapidly determining the cause of dyspnea or hypoxemia.

Perioperative patients and ICU patients are generally difficult to transport, and transport is associated with greater risk; rapid diagnosis of life-threatening respiratory symptoms such as dyspnea and hypoxemia is needed.

Point of care ultrasound is the ideal tool. Pulmonary ultrasound only requires a simple ultrasound instrument can be completed, most of the lung lesions will accumulate in the pleura, which can be detected by ultrasound.

The value of lung ultrasound in the diagnosis of interstitial syndrome were close to $\mathrm{CT}$ in sensitivity, accuracy and negative prediction [13].

In the diagnosis of interstitial syndrome by lung ultrasound, several signs such as pleural sliding sign, pulsating sign, B-line, consolidation and lung point should be recognized.

There are seven characteristics of interstitial syndrome [13].

This is a sign of a comet's tail. Originating from the pleura. Hyperechoic, similar to the pleural line, similar in appearance to lasers.
Replace the normal A line and extend to the bottom of the screen without attenuation.

It moves with the respiratory cycle of the lungs.

The presence of these characteristics is defined as B-line.

Multiple B-lines $(\geq 3)$ visible between the ribs are called lung rocket sign (Figure 4).

Interstitial syndrome is defined as the presence of diffuse lung rocket sign found by pulmonary ultrasound.

The disappearance of pleural slide sign and stratospheric sign and the discovery of lung points are typical pulmonary sonographic signs in clinical diagnosis of pneumothorax.

Although the above signs and the specificity of finding lung points for the diagnosis of pneumothorax is almost $100 \%$, however, it is not easy to find lung sites by ultrasound.

When pneumothorax is highly suspected, ultrasound should be used to scan each intercostal space to improve the detection rate.

There is a strong consistency between the assessment of pulmonary gasification degree by pulmonary ultrasound and chest CT.

Pulmonary ultrasound can provide real-time information about ARDS patients and provide visual evidence of lung ventilation at different stages of the disease, which can then guide treatment [14].

Perioperative period is one of the most important application fields of point of care ultrasound [4], point of care ultrasound can be used to achieve rapid assessment of cardiopulmonary function [10], emergency patients with satiety to identify, the author used point of care ultrasound to identify pulmonary edema early [12-14], early intervention, and then accelerate the recovery of patients; at the same time, the accurate identification of satiety can improve the safety of emergency operation.

Point of care gastric ultrasound has been used to evaluate gastric content and volume for about ten years, it can be used in adult, obese, pregnant, or paediatric patients whose gastric emptying might be delayed for perioperative assessment and and can be used to influence anaesthetic management [15]. As a new perioperative diagnostic tool, point of care gastric ultrasound needs to be characterized for anesthetist [15]. Most studies to date deal with validity considerations and suggest that point of care ultrasound accurately determines gastric volume [15-18]. Point of care gastric ultrasound can also be performed in a special group of patients with delayed gastric emptiness and gastric contents can be identified [15].

Peri-operative point of care ultrasound can diagnose life-threatening pulmonary lesions such as pulmonary edema, determine gastric content volume, and provide anesthesia safety. It can be seen that point of care ultrasound is a powerful perioperative tool to provide visual evidence for anesthesiologists and improve patient outcomes.

\section{Conclusion}

Point of care ultrasound is an important tool for perioperative diagnosis, perioperative patients change rapidly and require bedside ultrasound help achieve rapid diagnosis, 
can also evaluate the treatment effect, in view of hypoxemia or dyspnea BLUE protocol can be used, for shock RUSH protocol can be used, make the perioperative treatment to evidence-based medicine, the technology also should be the basic skills of anesthesia.

\section{Foundation Item}

Teaching Reform Project of Dalian Medical University in 2019 (DYLX2019016)

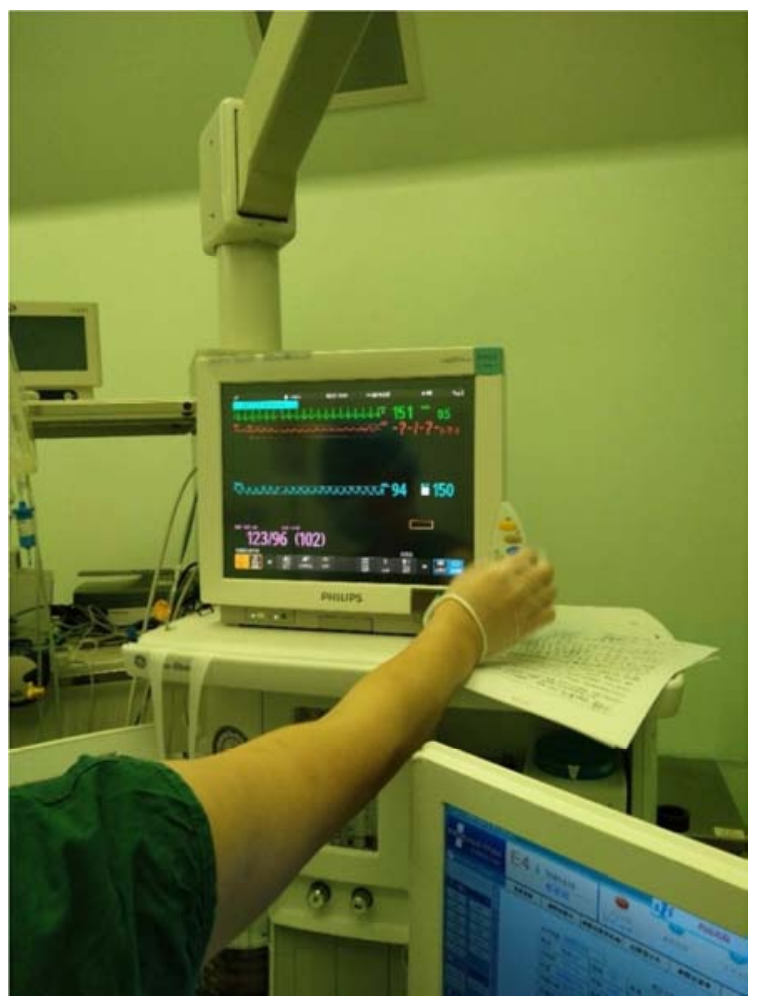

Figure 1. Preoperative vital signs

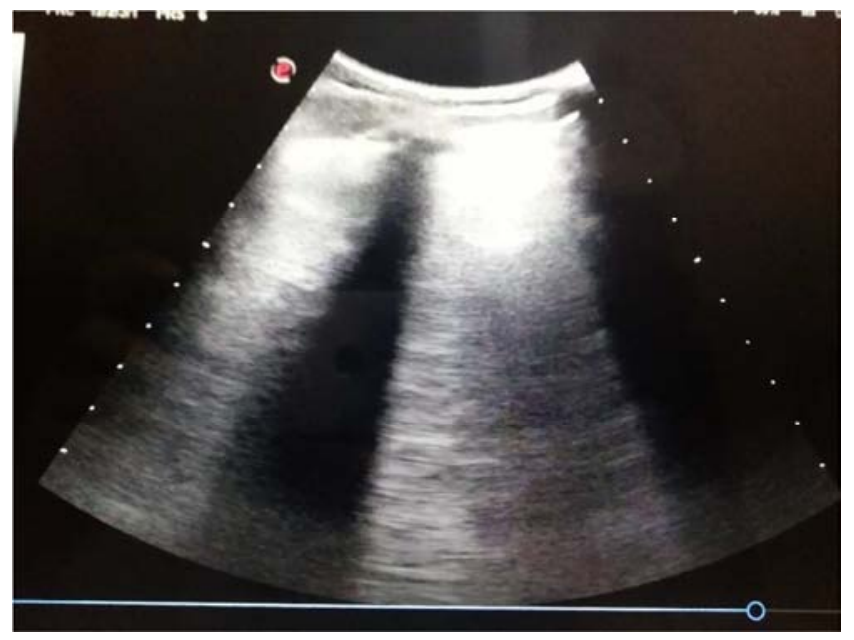

Figure 2. Lung ultrasound shows B-lines.

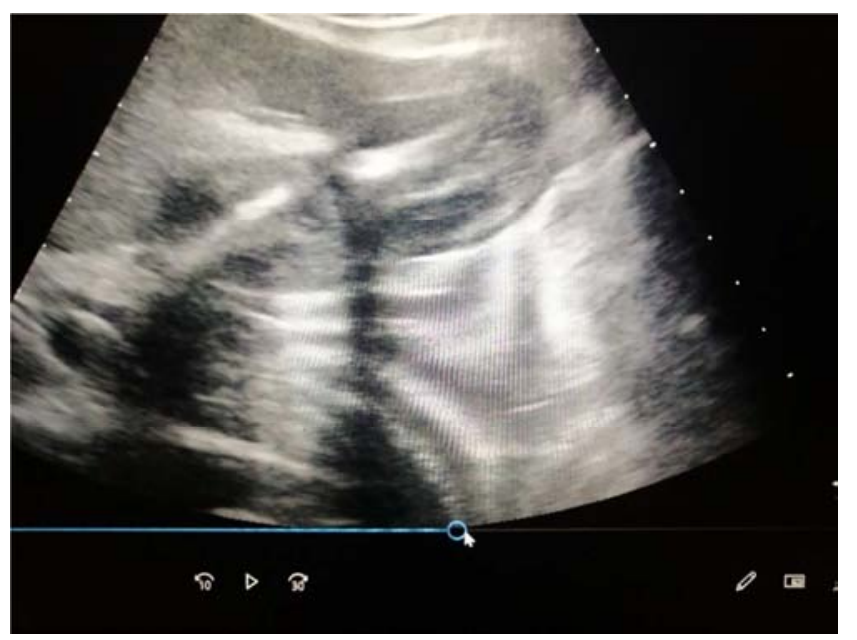

Figure 3. Gastric ultrasound showed a cross-sectional area of antrum was 3 $\times 3 \mathrm{~cm}$.

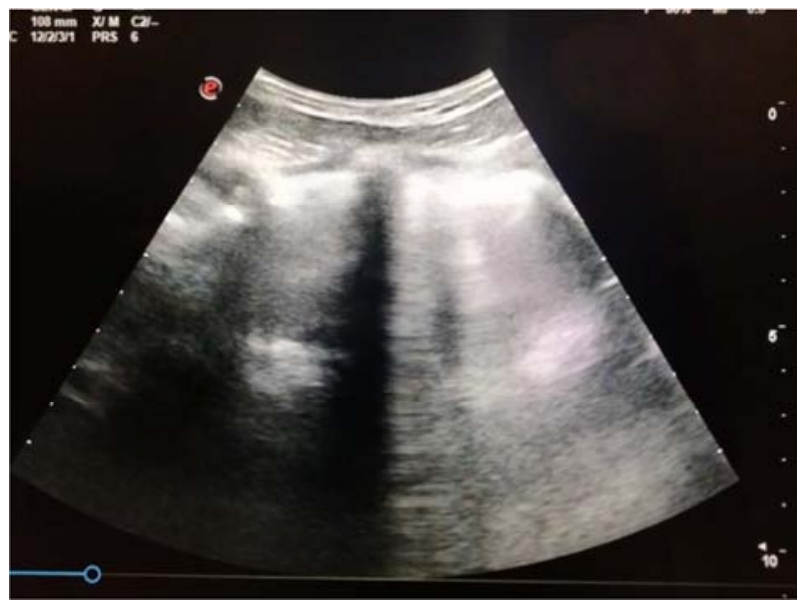

Figure 4. Multiple B-lines.

\section{References}

[1] YU Yuan, SANG You. (2017) Protocols of point of care ultrasound in critically ill patients. Chinese Journal of Diagnostic Electronics, 5 (3): 154-157.

[2] Zhao Hua, Wang Xiaoting, Liu Dawei, Zhang Hongmin, He Huaiwu, Long Yun. (2015) Effects of critical ultrasonic management of Peking Union Medical College Hospital on the etiological diagnosis of patients with acute respiratory failure. Chin J Medicine, 95 (47): 3843-3847.

[3] Shi Di, Xu Jun, Zhu Huadong, Yu Xuezhong. (2017) Analysis of expert consensus on clinical practice of emergency ultrasound in shock of unknown cause. Chinese emergency medicine, 37 (5): 394-396.

[4] Moore CL, Copel JA. (2011) Point-OC-Care Ultrasonography, N Engl J M, 364 (8): 749-757.

[5] Giovanni Volpicell. (2020) Lung Ultrasound B-Lines in Interstitial Lung Disease -Moving From Diagnosis to Prognostic Stratification. Chest, 158 (4): 1323-1324.

[6] P. Van de Putte, A. Perlas. (2014) Ultrasound assessment of gastric content and volume. British Journal of Anaesthesia, 113 (1): 12-22. 
[7] Yu Hong, Ze Peng, Yu Hai, Liu Jin. (2018) Perioperative ultrasound - the basic skill for anesthesists in new era. Journal of Clinical Anesthesiology, 34 (8): 814-826.

[8] P. Van de Putte, L. Vernieuwe, A. Perlas. (2019) Term pregnant patients have similar gastric volume to non-pregnant females: a single-centre cohort study. British Journal of Anaesthesia, 122 (1): 79-85.

[9] J. J. Moser, A. M. Walker, A. O. Spencer. (2017) Point-of-care paediatric gastric sonography: can antral cut-off values be used to diagnose an empty stomach? British Journal of Anaesthesia, 119 (5): 943-7.

[10] Peng Ge, Yalan Luo, Chukwuemeka Samuel Okoye, Haiyang Chen, Jiayue Liu, Guixin Zhang, Caiming Xu, Hailong Chen. (2020) Intestinal barrier damage, systemic inflammatory response syndrome, and acute lung injury: A troublesome trio for acute pancreatitis. Biomedicine \& Pharmacotheraphy, 132: $1-13$.

[11] Matthieu Jabaudon, ules Audard, Bruno Pereira, Samir Jaber, Jean-Yves Lefrant, Raiko Blondonnet, Thomas Godet, Emmanuel Futier, Céline Lambert, MSc; Jean-Etienne Bazin, Julie A. Bastarache, Jean-Michel Constantin, Lorraine B. Ware. (2020) Early Changes Over Time in the Radiographic Assessment of Lung Edema Score Are Associated With Survival in ARDS. CHEST, 158 (6): 2394-2403.
[12] Aron H. Ferreira, Antonio Pazin-Filho. (2020) Lung Ultrasound in a Patient With ARDS Secondary to Pancreatitis. CHEST, 158 (2): e85-e87.

[13] Danliel A. Lichtenstein. (2009) Lung Ultrasound in the Critically Ill.J Med Ultrasound, 17 (3): 125-142.

[14] Hatem S A, Marcelo H M. (2020) The Sound of Silence -The Power of Lung Ultrasound in the Interstitial-Alveolar Syndrome. JACC: CASE REPORTS, 2 (10): 1550-1552.

[15] K. El-Boghdadly, T. Wojcikiewicz, A. Perlas. (2019) Perioperative point-of-care gastric ultrasound. BJA Education, 19 (7): 219-226.

[16] D. Benhamou. (2015) Ultrasound assessment of gastric contents in the perioperative period: why is this not part of our daily practice? British Journal of Anaesthesia 114 (4): 545-8.

[17] Koenig SJ, Lakticova V, Mayo PH. (2011) Utility of ultrasonography for detection of gastric fluid during urgent endotracheal intubation. Intensive Care Med, 37: 627-31.

[18] Cubillos J, Tse C, Chan VW, Perlas A. (2012) Bedside ultrasound assessment of gastric content: an observational study. Can J Anaesth, 59: 416-23. 